\title{
Obstructive jaundice and severe pancreatitis due to the foramen of Winslow hernia with multiple anomalies
}

\author{
Neslihan Ekşi Bozbulut ${ }^{1 \oplus}$, İsmail Akdulum² ${ }^{2}$ Sinan Sar1 ${ }^{1}$, İbrahim Onur Özen ${ }^{3}$, \\ Yusuf Hakan Çavuşoğlu ${ }^{3}$, Öznur Boyunağa ${ }^{2}$, Aydın Dalgıç ${ }^{4}$, Buket Dalgıç \\ Departments of ${ }^{1}$ Pediatric Gastroenterology, ${ }^{2}$ Pediatric Radiology, ${ }^{3}$ Pediatric Surgery and ${ }^{4}$ General Surgery, Gazi University Faculty of \\ Medicine, Ankara, Turkey.
}

\begin{abstract}
Internal hernia through the foramen of Winslow is a very rare condition, especially in children. Here we report a 16-month-old girl who presented with obstructive jaundice and elevation of pancreatic enzymes and was ultimately diagnosed with internal hernia and malrotation by radiologic investigation and open approach surgery. To the best of our knowledge, obstructive jaundice with pancreatitis and other congenital abnormalities in children with the foramen of Winslow hernia have not been reported previously in the literature.
\end{abstract}

Key words: internal hernia, the foramen of Winslow, pancreatitis, obstructive jaundice.

Internal hernias are defined as protrusion of a viscus through a natural or secondary (postsurgical, traumatic, etc.) orifice of the abdominal cavity. ${ }^{1}$ The foramen of Winslow (Foramen epiploicum) is a passage between the greater (general peritoneal space) and lesser sac (omental bursa), allowing communication between these two spaces. ${ }^{1}$ Herniation of the intestines through this foramen constitutes only $8 \%$ of internal hernias. The rate of preoperative diagnosis is very low in these patients. ${ }^{2}$ Typical patients are middle-aged, with a sudden onset of severe abdominal pain as a symptom of acute intestinal obstruction. ${ }^{2,3}$ Rarely, patients present with jaundice because of the pressure of the herniated intestines on the common bile duct. ${ }^{1}$ It is a very rare condition in children and it is difficult to suspect this clinical situation in this age group..$^{2-4}$ Accompanying pancreatitis has not been reported before in children. Herein, we report a case of an infant with multiple congenital anomalies who presented with

Neslihan Ekşi Bozbulut

neslihaneksi@hotmail.com

Received 12th July 2018; revised 25th December 2018; accepted 29th December 2018 pancreatitis and obstructive jaundice secondary to a foramen of Winslow hernia.

\section{Case Report}

A 16-month-old girl who was previously healthy was admitted to our hospital with a onemonth history of restlessness, loss of appetite, abdominal pain, jaundice, and acholic stool. She had no pruritus. She had been hospitalized for 20 days in another clinic. Abdominal ultrasonography showed dilatation of intrahepatic bile ducts and ductus choledochus, and abnormal anatomic position of the head of the pancreas. For further examination, the patient was referred to our hospital.

On physical examination, her body weight was $7000 \mathrm{~g}(<3 \mathrm{p})$ and height was $73 \mathrm{~cm}(3-10 \mathrm{p})$. Her skin and sclera was icteric, spleen and liver were palpable 4 and $1 \mathrm{~cm}$, respectively. She had also dysmorphic facial characteristics (broad nasal root, long eyelashes, prominent forehead) and bilateral clinodactyly.

Blood tests revealed elevated transaminases, serum total bilirubin, conjugated bilirubin, amylase, and lipase (Table I). Millimetric 
Table I. Laboratory findings.

\begin{tabular}{lccc}
\hline Laboratory results & Admission & Postoperative (5th day) & Postoperative (one month) \\
\hline ALT (Range: 0-40 U/L) & $71 \mathrm{U} / \mathrm{L}$ & $68 \mathrm{U} / \mathrm{L}$ & $19 \mathrm{U} / \mathrm{L}$ \\
AST (Range: 0-40 U/L) & $152 \mathrm{U} / \mathrm{L}$ & $124 \mathrm{U} / \mathrm{L}$ & $42 \mathrm{U} / \mathrm{L}$ \\
GGT (Range: 0-50 U/L) & $810 \mathrm{U} / \mathrm{L}$ & $421 \mathrm{U} / \mathrm{L}$ & $36 \mathrm{U} / \mathrm{L}$ \\
Total bilirubin (Range: 0.3-1.2 mg/dl) & $15.3 \mathrm{mg} / \mathrm{dl}$ & $7.3 \mathrm{mg} / \mathrm{dl}$ & $0.94 \mathrm{mg} / \mathrm{dl}$ \\
Conjugated bilirubin (Range: 0-0.5 mg/dl) & $13.3 \mathrm{mg} / \mathrm{dl}$ & $6.3 \mathrm{mg} / \mathrm{dl}$ & $0.15 \mathrm{mg} / \mathrm{dl}$ \\
Amylase (Range: $28-100 \mathrm{U} / \mathrm{L})$ & $1376 \mathrm{U} / \mathrm{L}$ & $136 \mathrm{U} / \mathrm{L}$ & $57 \mathrm{U} / \mathrm{L}$ \\
Lipase (Range: $0-67 \mathrm{U} / \mathrm{L})$ & $1813 \mathrm{U} / \mathrm{L}$ & $107 \mathrm{U} / \mathrm{L}$ & $30 \mathrm{U} / \mathrm{L}$ \\
\hline
\end{tabular}

ALT: Alanine transaminase, AST: Aspartate transaminase, GGT: Gamma-glutamyltransferase.

echogenicity in the gallbladder lumen, dilatation of the common bile duct and intrahepatic bile ducts, and right pelvic ectopic kidney were observed on abdominal ultrasonography. Secundum atrial septal defect (ASD) was detected by transthoracic echocardiographic assessment.

Magnetic resonance cholangiopancreatography (MRCP) revealed dilatation of choledochus, common hepatic, and the intrahepatic bile ducts; structural abnormality of the pancreas (the head and the uncinate process extending into the hepatic hilum and upward to the lesser curvature of the stomach); splenomegaly; and intestinal malrotation (Fig. 1). Duodenojejunal junction and jejunal loops were located on the right side of the midline at upper gastrointestinal series. Computer tomography (CT) of the abdomen revealed more detail about the anatomy of the pancreas and vascular structures. Many dilated venous collaterals were observed at the confluence of the main portal vein and the splenic vein, which also covered the head of the pancreas, and the right portal vein narrowed at confluence. The hepatic artery arose from the celiac trunks and ran inferiorly, crossing the superior mesenteric
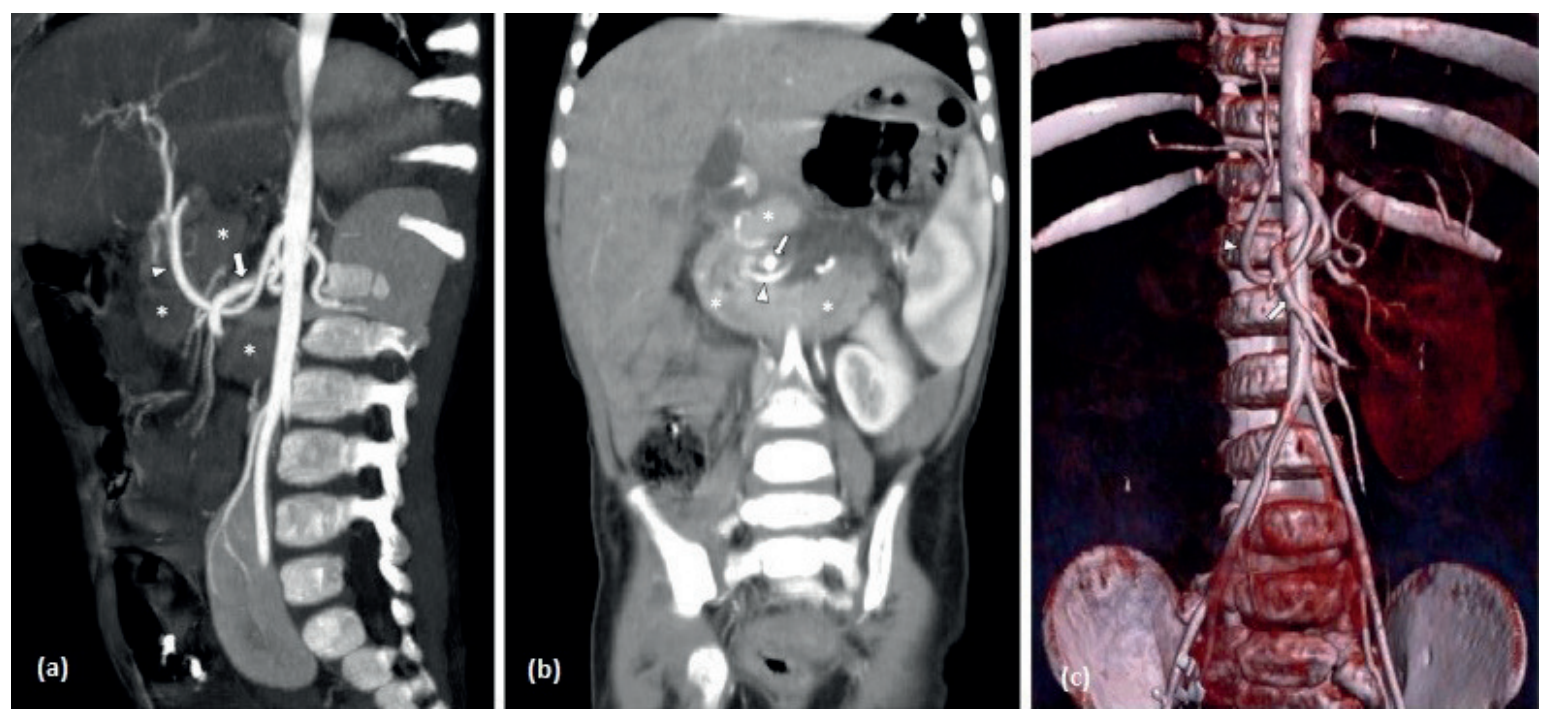

Fig. 1. Magnetic resonance cholangiopancreatography (MRCP) findings, sagittal oblique $5 \mathrm{~mm}$ maximum intensity projection (MIP) (a), $1 \mathrm{~mm}$ coronal multiplanar reformat (MPR) (b) and anterior view volume rendering technique (VRT) (c) images demonstrate superior mesenteric artery (SMA) (arrow) runs above hepatic artery (arrow head) and hepatic artery elongated. Pancreas $\left(^{*}\right)$ head and uncinate process shifted upwards and herniated through the foramen of Winslow. SMA proximal side is narrowed due to compression of herniated structures. 
artery inferiorly, reaching the liver by traveling parallel to the splenic vein, while the head of the pancreas was turned incompletely around the hepatic artery and celiac trunk (Fig. 1); prominent Wirsung channel was also seen. Posterior arcus fusion defect of the vertebra was detected at the $5^{\text {th }}$ lumbar and sacral vertebra on abdominal CT. Herniation of appendix, cecum, ascending colon, and a portion of the small intestine through the foramen of Winslow into the lesser sac, disrupted anatomic position of the pancreas (uncinate process pushing the stomach upwards), compression of the common bile duct by the duodenum, and malrotation were identified by open approach surgical exploration. Intestines were mobilized with traction behind the hepatoduodenal ligament. Position abnormality of the pancreas returned to normal after reduction of the intestines. External compression of the foramen of Winslow hernia on the biliary and pancreatic ducts caused obstructive jaundice and acute pancreatitis.

Her postoperative recovery was good with no complications. After surgery, her jaundice completely disappeared, and all laboratory abnormalities showed significant improvement (Table I). She was discharged from the hospital five days after the operation. One month after the surgery, laboratory results all returned to normal references. On the third month of her follow-up period, she had remained asymptomatic, abdominal ultrasonography revealed no abnormalities of the biliary tract and pancreas.

Informed consent of the subject was received from the family.

\section{Discussion}

The foramen of Winslow hernia accounts for $8 \%$ of internal hernias. It is seen much less frequently in children than in adults, and rare cases have been reported until now. ${ }^{2-4}$ Etiological factors might be an enlarged foramen of Winslow, mobile ascending colon caused by failure of secondary fusion of the colon with the posterior abdominal wall, common mesentery for the whole intestine, or a mobile small intestine resulting from abnormal length of the mesentery. ${ }^{1,4}$

The foramen of Winslow hernia is difficult to diagnose both clinically and radiologically. The most common clinical presentation of foramen of Winslow hernia is intestinal obstruction. Most of the reported pediatric patients in the literature presented with clinical findings of acute intestinal obstruction. ${ }^{2,4}$

Differently from other pediatric cases, our patient did not have serious abdominal pain or vomiting that suggested intestinal obstruction. Because of the separation of the lesser sac from the anterior abdominal wall, clinical findings of intestinal obstruction, even intestinal necrosis, might be overlooked in these patients. ${ }^{2}$ In some particular patients, obstructive jaundice was reported due to the direct compression of the bile ducts by the herniating viscus., ${ }^{3,5} \mathrm{We}$ observed obstructive jaundice and pancreatitis due to direct compression of the common bile duct because of the disrupted anatomic position of the pancreas and duodenum in our case. Similarly, an adult case was reported with obstructive jaundice and acute pancreatitis caused by herniation of the small bowel through the foramen of Winslow, like our case the common bile duct was compressed by the herniated bowel and believed that acute pancreatitis was due to this compression. ${ }^{5}$

The rate of preoperative diagnosis by clinical findings and laboratory abnormalities including imaging is very low, and most patients are diagnosed by laparotomy. "Narrowed portal vein" sign on CT imaging is considered to be a clue for the foramen of Winslow hernia, as seen in our case. ${ }^{6}$ Intestinal rotation anomalies were present in all pediatric patients. ${ }^{2-4}$ Our patient also has multiple congenital anomalies, atypical facial appearance, clinodactyly, right pelvic ectopic kidney, secundum ASD, and posterior arcus fusion defect of the vertebra, but we have not found a specific genetic reason for these congenital anomalies yet. 
In conclusion, foramen of Winslow hernia is a very difficult entity to diagnose, especially in children. Although acute intestinal obstruction is the most common presentation, different signs and symptoms, such as obstructive jaundice and pancreatitis, can be observed in these patients without intestinal obstruction as a symptom. We need to be careful and heighten awareness of its identification, both clinically and radiologically, in patients presenting with these findings.

\section{REFERENCES}

1. Martin LC, Merkle EM, Thompson WM. Review of internal hernias: radiographic and clinical findings. AJR Am J Roentgenol 2006; 186: 703-717.
2. Mboyo A, Goura E, Massicot R, et al. An exceptional cause of intestinal obstruction in a 2-year-old boy: strangulated hernia of the ileum through Winslow's foramen. J Pediatr Surg 2008; 43: e1-e3.

3. Antao B, Hamill J, Samuel M, Hiorns M, Pierro A. Herniation through the foramen of Winslow presenting as obstructive jaundice. Pediatr Surg Int 2005; 21: 560-562.

4. Zer M, Dintsman M. Incarcerated "Foramen of Winslow" hernia in a newborn. J Pediatr Surg 1973; 8: 325.

5. Joo YE, Kim HS, Choi SK, et al. Internal hernia presenting as obstructive jaundice and acute pancreatitis. Scand J Gastroenterol 2002; 37: 983-986.

6. Yamashiro T, Ikeda H, Fujikawa A, et al. Internal hernia through the foramen of Winslow: the "narrowed portal vein" sign on abdominal CT. Emerg Radiol 2013; 20: 247-250. 